\title{
Mass Thickness Measurement in TEM: A New Single Standard Method for Convenient Quantification by TEM EDS
}

\author{
P. Statham ${ }^{1}$, J. Sagar ${ }^{1}$, J. Holland ${ }^{1}$, J. Manktelow ${ }^{2}$, S. Lozano-Perez ${ }^{2}$ \\ ${ }^{1}$ Oxford Instruments NanoAnalysis, High Wycombe, Bucks HP12 3SE, U.K. \\ ${ }^{2}$ University of Oxford, Department of Materials, Oxford OX1 3PH, U.K.
}

The mass thickness, $(\rho \mathrm{t})_{\mathrm{sp}}$, of a thin film specimen is needed for absorption corrections in X-ray analysis and the equivalent areal density $\left(\right.$ atoms $/ \mathrm{m}^{2}$ ) for constituent elements is also useful. To measure thickness using EDS, we need a reference for the x-ray signal. Dijkstra et al [1] used a bulk target as a reference in TEM but the yield was too high at currents suitable for analysis of a thin sample. Boon [2] devised a beam current monitor linear over 3-4 decades so that a bulk target could be measured at much less current than the specimen and a beam current correction applied. Nevertheless, at TEM voltages (e.g. $200 \mathrm{kV}$ ) absorption corrections are severe for bulk material and the method still requires the use of many standards. Watanabe's zeta factor method [3] requires thin film standards of known mass thickness and composition and a method of measuring the beam current. However, beam current measurement is not always available, convenient or of sufficient accuracy and there is a dearth of suitable thin film standards.

Here we consider a new method that uses a single reference standard and can be used on any TEM. The procedure involves recording an X-ray spectrum from the reference standard before each session of acquisitions on a real specimen. There is no need to measure the beam current; it just needs to be stable for the duration of the session. For a thin specimen with uniform ionization throughout, the counts recorded for the emission line of element A can be written as

$$
I_{A}=(\rho t)_{s p} \cdot C_{A} \cdot\left[N_{0} / A_{A} \cdot\left(Q_{A} \cdot f_{A}\right)\right] \cdot f(c h i)_{A} \cdot D_{s p} \cdot(\Omega /(4 \pi)) \cdot \varepsilon_{A}
$$

for element mass fraction $\mathrm{C}_{\mathrm{A}}$, Avogadro constant $\mathrm{N}_{0}$, atomic weight $\mathrm{A}_{\mathrm{A}}$, ionization cross section $\mathrm{Q}_{\mathrm{A}}$, fractional emission $\mathrm{f}_{\mathrm{A}}$, self absorption $\mathrm{f}(\mathrm{chi})_{\mathrm{A}}$, electron dose $\mathrm{D}_{\mathrm{sp}}$, detector solid angle $\Omega$ and conversion efficiency $\varepsilon_{\mathrm{A}}$. The terms in square brackets can be calculated from theory, as in previous methods for TEM quantitative analysis and $\mathrm{f}(\mathrm{chi})_{\mathrm{A}}$ can be evaluated if the composition and mass thickness are known. If we have a reference standard where $(\rho \mathrm{t})_{\text {ref. }} \mathrm{C}_{\text {ref }}$ is known and an X-ray detector that has been characterized so that conversion efficiency $\varepsilon$ is known as a function of energy [4] then we can determine ( $D_{\text {sp }} \cdot(\Omega /(4 \pi))$ ) from the measurement on the reference standard provided we use the same beam current and $\mathrm{kV}$. When a spectrum is recorded from the specimen, equation (1) can be then be used to determine $(\rho t)_{\text {sp. }} C_{A}$ for each element. $(\rho t)_{s p}$ is then obtained by assuming that the mass fractions sum to unity and iteration can be used to refine $\mathrm{f}(\mathrm{chi})_{\mathrm{A}}$. Doing a "beam measurement" on the reference standard at the start of any session thus replaces explicit measurement of beam current.

To make this technique accessible requires a suitable well-characterized reference standard to be available. Silicon nitride TEM support films within a standard $3 \mathrm{~mm}$ diameter disk are now widely available and can be manufactured to have highly uniform thickness over a large area. Although film composition, thickness and density is nominal, we have characterized some samples in an SEM at $30 \mathrm{kV}$ using a transmission holder and pure bulk reference standards so that we can measure $(\rho t)_{\text {ref. }} C_{\text {ref }}$ at a series of points all over the support film. An example shown in Fig. 1 shows that the mass thickness 
estimate is reproducible across a region spanning $0.6 \mathrm{~mm}$. In a TEM, the detector is typically positioned very close to the sample and the sample holder may cause variable occlusion of the available solid angle $[5,6]$. Solutions like tilting the holder as shown in Fig.2 and removing material on the side of the holder facing the detector as in Fig. 3 can help alleviate the occlusion [5,6]. Any occlusion alters $\Omega$ in equation (1) and thus influences any method of mass thickness measurement but the reference sample can be used to test what area on the sample is safe to use for measurement. With a 5 degree specimen tilt we have been able to obtain consistent mass thickness measurements in a JEOL JEC 2100 TEM over a region spanning $0.8 \mathrm{~mm}$ (Fig.4). We are currently validating the reference standard for durability and refining the method for characterization before we move on to testing the accuracy and reproducibility on known materials. The new method should allow mass thickness measurements to be obtained routinely in any TEM instrument with reasonably stable beam current, with no need for a beam current meter. The new reference standard also allows the available region on the specimen, for valid measurements without occlusion, to be determined.

\section{References}

[1] J.M. Dijkstra et al (1994) Microchimica Acta 114/115, 277-284

[2] G. Boon (2000) Thesis, ISBN 90-386-2781-5

[3] M.Watanabe and D.B.Williams (2006) J.Microscopy 221, Pt 2, 89-109

[4] P.J. Statham (2010) Microsc. Microanal.16 (Suppl. 2) 1304-1305

[5] N. Rowlands, E.F. Schumacher and A.W. Nicholls (2013) Microsc. Microanal. 19 (Suppl 2), 1074-5

[6] N. Zaluzec (2014) Microsc. Microanal. 20, 1318-1326
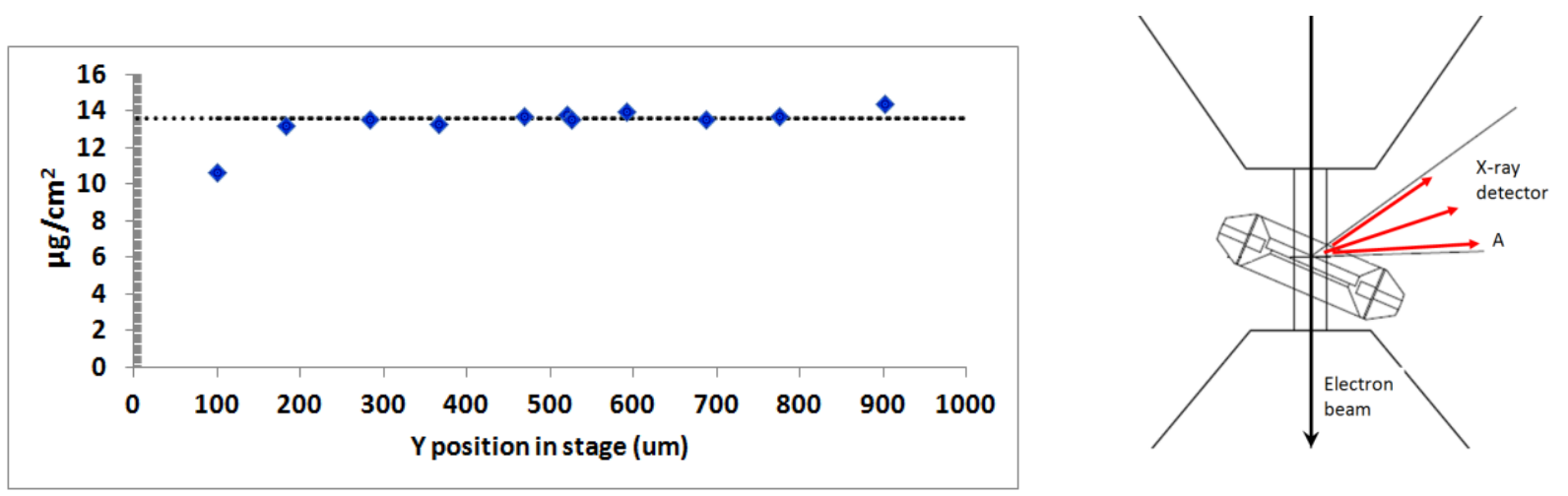

FIG. 1. Si mass thickness measurements of reference standard in SEM 30kV

FIG. 2. Specimen geometry showing

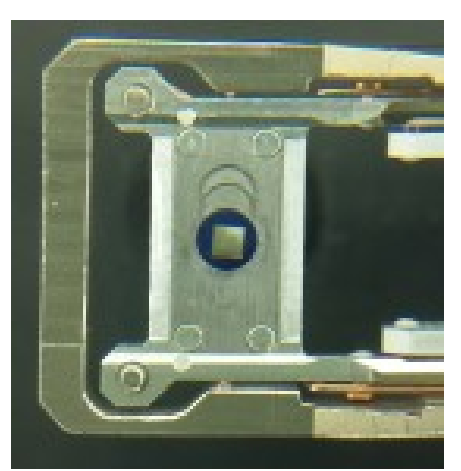

FIG. 3. Low background TEM holder showing cut out on side facing detector above potential for occlusion of rays A by holder

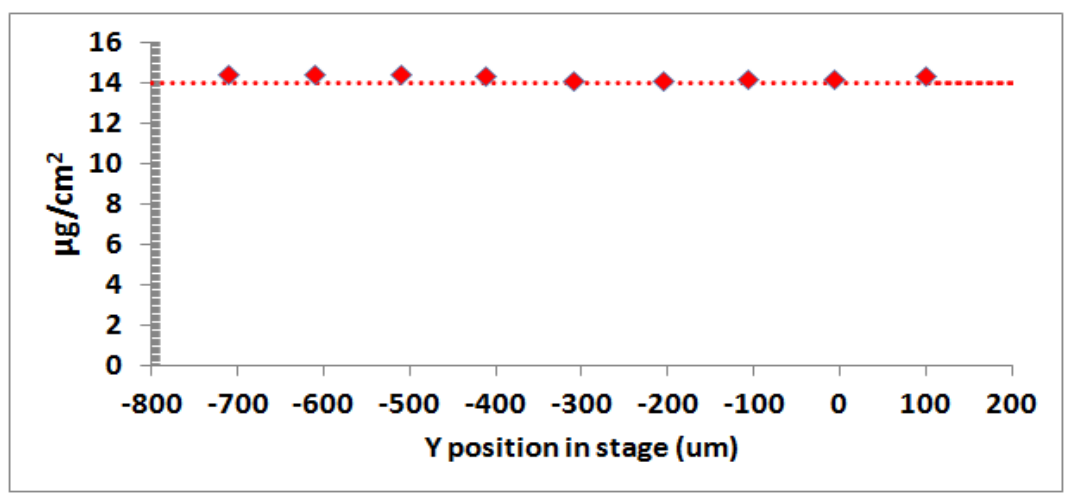

FIG. 4. Mass thickness measurements $5^{\circ}$ tilt, JEC 2100 TEM $200 \mathrm{kV}$ 